Es ist wirklich Zcit, mit cincr genauen quantitativen Untersuchung der Harnsedimente unter dein Mikroskop zu beginnen, wozu vor allen Dingen notwendig ist, eine Größeneinheit des Präparats und die Mes. sung des Gesichtsfeldes einzufülıren. Die Bedeutung einer quantitativen Bestimmung der Harnsedimente für die Beurteilung des Charakters und des Verlaufs der Nierenerkrankungen ist unstreitig; jedenfalls ist sie nicht gerikger - wie mir scheinen will, ist sie sogar noch größer - als die anderer quantitativer Bestinımungen, wie z. B. der Körpertemperatur, der Pulsfrequenz, der arteriellen Druckhöhe, der Menge der Elythrozyten, Leukozyten und des Hämoglobins inı Blut oder des spezifischen Gewichts, der Zucker- und Eiweißmenge des Harns. Es ist ja sehr wahrscheinlicl, daß dic Beobachtung größtmöglicher Gellauigkeit in der quantitativen Analyse der Harnsedimente bald zur Beleuchtung wenigstens ciniger dunkler Fragen auf dem Gebiet der Nierenkrankheiten (z. B. der Pharmako- und Diätotherapic der Nephri tiden) führen könnte; vor allen Dingen aber würde es mit Hilfe systemnatischer Analysen möglich sein, den Verlauf der Krankheit zu verfolgen. Ehe ich die Idee einer mehr oder minder genauen quantitativen mikroskopischen Analyse der Harnsedimentc näher erörtere, nö̈chte ich z we zu diesen Zweck von mir vorgeschlagene $Z$ äll lk a 11 ne rn besehreiben, welche geschlossene Räume von gennu bestinumtem Volumen clarstellen, dic eine allseitige $\mathrm{Be}$ -

\section{Zur Methodik der Zählung organisierter Harnelemente.}

\section{Von A. Kakowski in Kiew.}

Die mikroskopische Untersuchung der Harnsedimente befindet sich bis auf den heutigen T'ag in cinein ganz elementaren Zustande; zwar ist es um die qualitative Analyse noch befriedigend bestellt, allein die Versuche einer quantitativen Bestimmung des Harnsediments laalten einer Kritik nicht stand.

Gewöhnlich schreibt man: ,rcichlicher oder geringer Niederschlag, 3-5 Zylinder im Präparat, 10-15 Erythrozyten im Gesichtsfelde" etc. Abcr diese Angaben druicken deshalb schon nichts aus, weil es an allgeinein anerkannten und akzeptierten Einheiten (z. B. einer Größeneinheit des Präparats) mangelt. Der eine versteht unter einem Präparat eine Fläche von $100 \mathrm{qmm}(10 \mathrm{~mm} \quad 10 \mathrm{~mm})$ bei einer $0,1 \mathrm{~mm}$ dicken Sclicht, der andere eine $400 \mathrm{qmm}(20 \mathrm{~mm} \times 20 \mathrm{~mm})$ große, $1 \mathrm{~mm}$ dicke Schicht. Wic wir sehen, verhalten sich diese Präparate der Größe nach wic $1: 40, \mathrm{~d}$. h. daß der Ausdruck ,5 Zylinder im Präparat“" im crsten Falle nur $\tilde{5}$, im zweiten aber 200 Zylinder zu bedeuten hätte. Iie: tatsächliche Ziffer bleibt unbekannt.

Ein zweites Beispicl: „10 Erythrozyten im Gesichtsfelde." Nimmt man ein Ciesichtsfeld - sagen wir - bei Reicherts Ok. IV und Objekt. $7 \mathrm{a}$ als Flächeneinheit an, so wird ein Gesichtsfeld bei Ok. II und Objekt. 4b etwa 20 solcher Einhciten enthalten; fügen wir dazu noch cine eventuelle Differenz in der Dicke des Präparats wie zwischen 1 und 10, so ergibt sich ein Verhältnis der Gesichtsfelder von 1 : 200. Der Ausdruck ,10 Erythrozyten in Gesiehtsfelde“ ist also in einem Fallo als 10, im anderen als 2000 aufzufassen. Soll außerdem noch anvernoinnien werden, daß in crsten Fallc $0,1 \mathrm{ccm}$, in zweiten aber 1,0 ccm Sediment vorhanden war, so wächst der Unterschied in der Zahl der Elemente noch ins Zehnfache, und es ergibt sich eine ungeheure Differenz, nämlich wic zwischen 10 und 20000 .

Diese beiden Beispiele zeigen klar und deutlich, zu welchen groben Fehlern man gelangen kann, wenn man aus den Zalilenangaben der jetzigen Analysen logiseh richtige Schlüsse zieht.

Schließlich muß auch noeh erwähnt werden, daß sehr häufig zu wiederholten Analysen Harnportionen ganz verschiedener Beschaffenheit gelicfert werden und daß die Menge der Elemente in den Analysen cinmal im Gesichtsfelde, ein anders Mal im Präparat angegeben wird, ohne daß dabei bemerkt wird, wic groß die betreffenden Flächen des Gesichtsfeldes und des Präparates gewcsen sind. In der Tat ist niemand instande, ohne spezielle Berechnung anzugeben, wievicl Gesichtsfelder im Präparat enthalten sind, selbst wenn die Fläche des letzteren und dic Nummern des Objektivs und des Okulars genau bekannt sind.

Daher können die Angaben der bisher ausgeführten Harnanalysen auch nur zur Diagnose der Nephritis dienen, während sie zur Beurteilung des Verlaufs der Krankheit unter verschiedenen veränderlichen Bedingungen absolut unzureichend sind. Ebenso ist es unlogisch, auf Grund eines Vergleichs zweier oder mehrerer, in verschiedenen Laboratorien ausgeführten Analysen irgend welche Schlüsse ziehen zu wollen, wie es auch schwierig ist, wissenschaftliche Arbeiten zu verstehen, welche auf so ungenügenden mikroskopischen Harnuntersuchungen begründet sind.

Wie aus den oben angeführten Beispielen ersichtlich ist, hängt der große Unterschied in den aus den Zahlen der betreffenden Analysen gezogenen Schlüssen hauptsächlich nit der unbekannten und schwankenden Größe des Präparats und zunı Teil des Gesichtsfeldes zusammen. trachtung unter deir Mikroskop gestatten und mit Harnsediment gefüllt sind. (Siche Figur A.)

Apparat A stellt eine Kamnier dar, deren runde Grundfläche

$1 \mathrm{qcm}$, und deren Tiefe $1 / 10$ inm beträgt, sodal also der Rauminhalt $1 / 100$ ecin $(10 \quad \mathrm{cmin})$ ausmacht. Wie aus der die Kammer von oben darstellenden Zeichnung ersichtlich ist, besteht der Apparat aus folgenden 'T'eilen: dd ist der Objektträger in dessen Zentrum eine
Fig. A.

farızählkanmer A von uben. $2 / 3$.

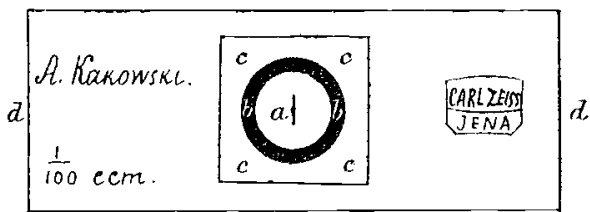

Der Strich-mit Finteilung in $1 / 10$ und $1 /$ ino mm Zwanzigfache Vergrößerung.

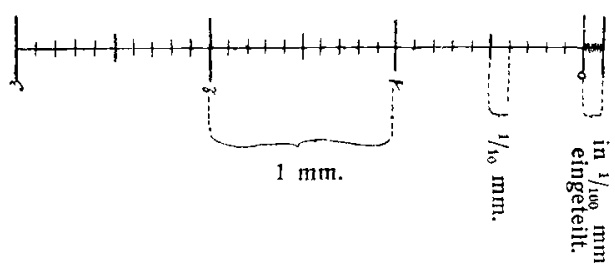
$1 \mathrm{qcm}$ Fläche messende, sehr sorgfältig geschliffene, runde Glasscheibe a (Durchmesser 11,284 inın) angeklebt ist. Diese Scheibe bildet den Boden der Kanmer, auf welchem ein $3,1 \mathrm{~nm}$ langer Strich mit Einteilungen von $1 / 10$ und $1 / 100$ mm eingraviert ist. Etwa $2 \mathrm{~mm}$ vom Rande des Kammerbodens entfernt ist cin gut geschliffener Glasrahmen ccec angeklebt. Zwischen Scheibe a und Rahmen ccec bleibt naturgemäß cine etwa $2 \mathrm{~nm}$ breite ringförmige Rinno $\mathrm{bb}$, als deren Boden der Objektträger dient. Der Rahmen ecec ist genau um ${ }^{1} / 10 \mathrm{~mm}$ dicker als die Scheibe a; bedecken wir die Kamner aber völlig gerade mit einem geschliffencn, unbiegsamen und zu diesem Zweck ziemlich dicken Deckglächsen, so erhalten wir einen geschlossenen Rauı von $1 \mathrm{qcm}$ Fläche und $1 / 10 \mathrm{~mm}$ Höhe, der von planparallelen Flächen, dem Deckgläschen von oben und der Scheibe a von unten begrenzt ist. Dic Rinne bb, welche Scheibe a einfaßt, dient nur zur Entfernung des überschüssigen Sediments und zur Verhütung seines Eindringens zwischen Deckgläschen und Rahmen ece, da die Tiefe der Kamıler dabei natürlich vergrößert werden würde.

Dicse Zählkammer mit cinem genau bestimmten $R$ auıninlıalt von $1 / 100$ ec n oder $10 \mathrm{cmm}$ (100 qinm Fläche und $1 / 10$ min Tiefe) würde ich vorschlagen normale Harnzählka $n$ mer zu nennen und als Präparateinheit anzunehmen. Die auf dem Kammerboden markierten Einteilungen in Hundertstel und Zehntel Millimeter können zu verschicdenen Messungen unter deln Mikroskop, hauptsächlich aber zur genauen Messung des Radius des Gesiclitsfelde s bei allen möglichen Linsen. systemen des Mikroskops dienen, was zur Bestimmung der Fläche des Gesichtsfeldes uncntbehrlich ist. Hat man nach Formel $\pi r^{2}$ die Größc der Fläche des zur Untersuchung bestimmten Gesichtsfeldes berechnet und dividiert man dann 100 q $1 n$ m durch die erhaltene Zahl, so ergibt sich, wicviel solcher Gesichtsfelder in einer Präparateinheit enthalten sind. Durch Multiplikation der in Gesichtsfelde gefundenen Zahl der Elemente nit dieser Ziffer erhält man dann die Anzahl der Elemente in einer Präparateinheit.

Die $\mathrm{H}$ a u ptmänge l der normalen Zählkammer A bei beständiger Arbeit bestehen in folgendem: Erstens können die Teilstriche auf dem Boden der Kammer durch Kristalle verdorben und durch Sediment verdunkelt werden; zweitens ist die für Zylinder ${ }^{1}$ ) und Formelemente völlig ausreichende Tiefe der Kammer für Kristalle zu gering, und drittens

1) Ueber $1 / 15 \mathrm{~mm}$ dicke Zylinder sind sehr selten anzutreffen. 
(rschwert die geringe Dicke des Präparats das Auffinden von Elcmenten, wonn solehe in nur geringer Zahl vorhanden sind.

Aus diesen Erwägungen heraus enpfelıle ich einen billigeren ${ }^{1}$ ) zweiten Apparat ohne Einteilungen auf den Kammerboden. Siehe Figur B I: a, -- eine runde Glasscheibe von einer 4 qcm großen Fläche und einem Durchmesser von 22,567 mm - ist der Bodell der Kamıncr, $b_{1} b_{1}$ die Rinne und $c_{1} c_{1} c_{1} c_{1}$ der Glasrahnien. Die Tiefe der Kamnier beträgt $1 / 4 \mathrm{~mm}$, da der Rahmen $\mathbf{c}_{1} \mathbf{c}_{1} \mathbf{c}_{1} \mathrm{c}_{1}$ um $1 / 4$ mun dicker ist als Scheibe $\mathrm{a}_{1}$ Dicsen Diniensionen der Kammer entsprecliend, nußß der Objektträger etwas größer sein. Das Volumen der Ka mmer B beträgt $1 / 10$ ec in oder 100 cmn $(400 \times 14)$, ist also zelmmal größcr als clas d(cr Kammer A, war für dic Untersuchung und Zällung von großcm Nutzen ist.

Figur B II stellt dieselbe Kamner in Profil (lar. $\left.{ }^{2}\right)$

Fig. BI.

Harnzählkammer B von oben. $\% / 3$.

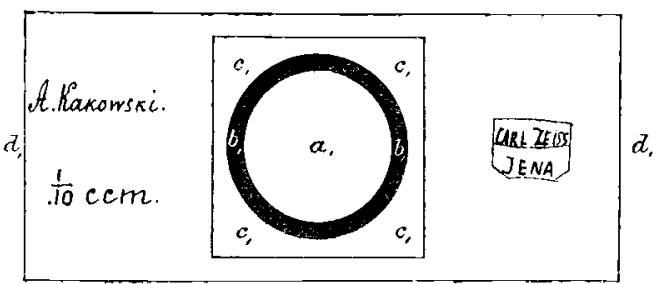

Fig. B II.

Jarnzählkammer B in Profil.

Doppelte VergröBerung.

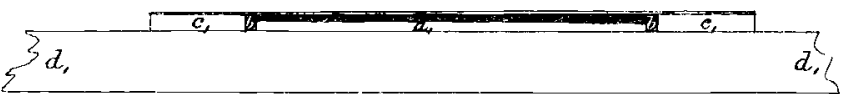

Prinzip der Zällung organisierter Elemente des pathologischen Harns. Mit Hilfe der von mir vorgeschlagenen Zählkammern läßt sich dic Menge der organisierten Elemente in einer Präparateinheit leicht feststcllen. Mit einer solchen Zählung allein darf inan sich abcr niclit begnügen, denn bci einem Patienten können siclı zehn Zylinder im Präparat befinden, das Sediment reicht abcr nur für zwci Präparatc aus, sodaß im ganzen 20 Zylinder im Sediment vorhanden sind, bei einem andern aber kann ein Zylinder im Präparat gefunden werden, es können dafür aber hundert solcher Präparate, im ganzen Sedimente also $100 \mathrm{Zy}$ linder vorhanden sein. Die absolute Zylinderzahl würde also im zweiten Falle in Wirklichkeit fünfınal größer sein als im ersten, während doch auf Grund der bloßen Zählung nach denı Präparat im crsten Falle zehnmal mehr Zylinder vorhanden wären als im zweiten. Hieraus geht deutlich hervor, daß zur genauen Zählung der organisicrten Harnelcmentc unbedingtes Erfordernis ist zu wissen, wieviel Präparateinheiten im betreffenden Scdiment enthalten sind und wie groß die Zahl der Wlemente im ganzen Sedimente ist. Darum muß das Scdiment jedesmal genau in Kubikzentimetern gemessen werden; Termini wic „reichlicher, geringer, mäßiger, flockiger Niederschlag“ u. a. sind, da sie als Maßausdruck nicht dienen können, auszuschalten.

Die Gewinnung des Sediments. Zu spcziellen Zwecken darf nur das Sediment solcher Harnportionen aufgefangen werden, welche geradc interessieren. $\mathrm{B}$ i der Beobachtung Kranker im allgemeinen wärc es am zutreffendsten, wenn man das Sedinient des ganzen 24 st ündigen Harns auffinge, wobei es zur Verhütung einer unter dem Finfluß von Mikroorganismen möglichen Harnzcrsetzung ratsall ist, Kampfor, Formalin oder 'Tlyymol zuzusetzen. Zur qualitativen nikroskopischen sowohl als auch zur chemischen Analyse ist unbedingt 24stündigc:r Harn zu verwcnden, zur systematischen quautitativen Bestimmung der Zylinder und Fornelemente ist er aber nicht tauglich, da (r) inınerlin leicht verdirbt und trübc wird, was cinen vollständigen Zerfall eines 'Teils dor Zylinder und Formelemente zur Folge hat; außerdem wird eino genaue quantitative Untersuchung durch eine große Mengo körnigcn Eiweißzerfalls, durch ausfallende Mineralbestandteile u. a. schr gehindert, ebenso wic eine T'rübung das Absetzen, Absondern und Zentrifugicren des Sediments beträchtlich ersclıwert. Endlich muß auch noch in Betracht gezogen werden, daß eine systeniatische quantitative Untersuchung des Sediments nur zum Zweck des Vergleichs der verschiedenen Analysenresultate angestellt wird, aus welchem Grundc es wichtig ist, nur unter völlig gleichen Bedingungen von den Nieren abgesonderten Harn zu nehmen. Deshalb bestehe ich eben auf Morgenharn, der abgesehcı von dem Vorzug der Frische noch den Vorteil bietet, daß er - was Ennähung, Ruhe, Schweißabsonderung etc. des Patienten betrifft -- gewölınlich untcr den gleichen Bedingungen von den Nieren

1) Aus Sparsanıkeitsgründen entschloßich mich, eine runde Kammer machen zu lassen, obgleich eine viereckige zur Zählung weit bequemer wäre. - ${ }^{2}$ Zweckmäßig ist es, sich außer diesen beiden Kammern woch eine kleine Kammer A ohne Teilungen anzuschaffen. abgesondert wird, und alle dicse Bedingungen übcn doch bekanntlich einen großen Einfluß auf dic Eigensclaften des Harns aus. Zu keiner anderen Zeit kann der Patient unter so gleichförmige Bedingungen gestellt werden, wic das des Nachts bequenı geschelıen kalın, was für clic wissenschaftliche Beobachtung äußerst wichtig ist. Der Patient nuls daran gewöhnt werden, einige Stunden vor dem Einsclılafen sein Nachtinahl einzumelinien und bis zum Morgen weder zu essen noch zu trinken. ${ }^{1}$ ) Kann der Patient in der Naclit nicht schlafen, so darf er docll keinesfalls das Bett verlassen. Temperatur und Feuchtigkeitsgrad der Zinmerluft müssen gleich scin. Vor dem Einschlafen entleert der Patient die Blaso und hält dann nacl Möglichkeit den Harn in der Blasc bis zum Morgen zurïck (was in Interesse der Frische des Harns geboten ist), an Morgen uriniert er dann unmittelbar in ein sauberes Spitzglas. Ist cler Patient nicht imstande, den Harn voll Abend bis zum Morgen zurückzuhalten, so muß jedenfalls sein Naclit- und Norgenharn aufgehoben worden. Kurz, zu systcmatischen vergleichenden Analysen des Sedinients mu meines Erachtens frisclier Harn verwandt worden, der voll den Nieren im Laufeder aclit, für den Sclilaf bestionten Stunden abgesondert wird.

$\mathrm{Zum} \mathrm{Zwcek} \mathrm{des} \mathrm{Absetzens} \mathrm{wird} \mathrm{der} \mathrm{Harn} \mathrm{am} \mathrm{besten} \mathrm{in} \mathrm{cin}$ lolıcs, $250-300$ ccul fasscndes Spitzglas nit Einteilung(n zu $50 \mathrm{com}$ gegossen. Zum Absetzen können verschiedene Harnincigen, jc nacl dem in Harn enthaltenen Sodiment, genommen werden; am richtigsten ist es, den ganzen Nacht- und Morgenharn dazu zu verwenden. Das Spitzglas wird bedeckt, und nun muß sich der Harn an einem kühlen Ortc absetzen, wozu erfahrungsgemäß im allgcmeincn fünf bis seclis Stunden genügend sind.

Des weiteren nuuß zur Konzentrierung das ganze Sediment (ntweder mit einer Pipctte aus dem Spitzglase genommen oder einfacl in nach Kubikzentimetern graduierte, mit Untereinteilungen (von Zehnteln) versehene Probiergläschen gegossen und in diesen zentrifugiert werden. Gut wäre es, das Sediment stets bei bestiminter Geschwindigkeit und in derselben Zeit, $z$. B. jedesmal innerhalb fünf Minuten, zu zentrifugieren. Es ist aber nicht gerade durchaus notwendig, in dieser Bezichung peinlich genau zu sein; denn ist das Sediment dünncr, so wird unter sonst gleichen Bedingungen sein Quantum größer scin, sodaß dic Genauigkeit der Zählung nicht beeinträchtigt und die absolut Zahl der Elcmente inı ganzen Sediment in beiden Fällen dieselbc scill wird. Zur bequenıeren Untersuchung muß uıı so länger zentrifugiert werden, je dünner das Sediment, d. h. je weniger Elcmente und je inelır Schleim darin ist.

Die Menge des Sediments ist in Kubikzentinictern anzugeben und zur bequemeren Vergleichung auf $100 \mathrm{ccm}$ Harn $\mathrm{zu}$ berechnen, welches Quantum ich als Sedimenteinheit anzunehmon vorschlage. Die Zalıl 100 wählte ich der Geläufigkeit wegen und vor allen Dingen, weil sie der bei jedem Nephritiker während einer Nacht aufzufangenden Harnnenge entspricht. Die die Menge des Sediments in $300 \mathrm{ccm}$ Harn ausdrückende Zahl muß natürlich durch drei geteilt, die den Niederschlag in $50 \mathrm{ccm}$ angebende doppelt genommen werden; kurz, die aus eincm beliebigen Harnquantum gewonnenc Menge des Sediment mu $\beta$ auf eine Einheit, d. h. auf das in 100 cen Harn enthaltenc Sedimentquantum, zurückgefülrt werden und ist nur in Kubikzentimetern zu rcgistrieren, ohnc daß angegcben zu werden braucht, aus welcher Harnmenge sie gewonnen ist, da sich das von selbst versteht. Nach der Registrierung wird der überstelıendc Harn aus dem Probierglase ausgegoss $\left(n^{2}\right)$ und das Scdintent in demselben Glasc vor sichtig geschïttelt, damit dic Zylinder nicht zcrbrechen, worauf es zur quantitativen Bestimnung sciner organisierten Bestandteile fertig ist.

Die Zällung der organisierten Elemente des Sediments nit Hilfe der Harnzählkammern. Zur Zählung muß bekannt sein: 1. dic Menge der jeweiligen Gesichtsfelder in eincr Präparatcinheit, 2. dic Größc des Präparats, 3. das Quantum des zu untersuchenden Sediments und 4. dic Zahl der Elementc in Gesichtsfelde des Mikroskops. Alsdann läßt sich durch cinfache Multiplikation dic genaue Zahl der Elcnicnto im ganzen Sediment selir rasch bestimmen.

1. Dic erstc Frage läßt sich mit Hilfe von Teilungen der normalen Harnkan mer lösen, welche es el möglichen, den Durchmesser eines beliebigen Gesiclitsfeldes in Hundertsteln Millimetern genau und in Tausendstcln ungefähr $\mathrm{zu}$ bestion inen, da dic kleinstc Abteilung des Kammerbodens ${ }^{1} / 100 \mathrm{~mm}$ beträgt. ${ }^{3}$ ) Hin groß3cr Vorzug dieser Teilungen besteht darin, daß zu den Harnanalyson jedes belicbigc Mikroskop, gleichvicl welcher Firma oder welches Systems gebraucht werden kann. Ist der Durchmesser, und

1) Ist sehr starker Dirst vorhanden, so solI jede Nacht dassc:He Getränk in den gleichen Quant:täten eincenommen werden

2) Fin bestimmtes gcringes Quantum Harn roll doch über de.m sichtbaren Sedinient stets bleiben.

3) Di: Zählkammern der Blutkörperchen (Thoma-Zeiss u. a sind zur Harnuntersuchung ungeeignet, weil die kleinste Finteilung $1 / 20 \mathrm{~mm}$ ausinacht und der $K$ amnierboden unter $1 \mathrm{~cm}$ groß jst; sic können nur im höchsten Falle zur ungefähren Messung des Radius des Ge. sichtsfeldes dienen. 
somit auch der Radins des Gesichtsfeldes bekannt, 'so ist nach Formel 100

$\pi \mathbf{r}^{2}$ leicht zu bestimmen, wieviel solcher Gesichtsfelder in Präparat

cullialten sind, da eine konstante Ǵröße ist, näınlich 3,14159

Zur Erleichterung dieser Berechnungen gestatte ich mir hier die Anf stelling ciner kurzen Tabelle, wclclic genau anzeigt, auf welche Durchmesserlänge das Mikroskop eingestellt werden mu $\beta$, soll in ganzen Zallen die Zalll der Gesichtsfelder in einer l'räparateinlıeit, d. l. in cinenı Quadratzentimeter, crlalten werden.

\begin{tabular}{|c|c|c|}
\hline $\begin{array}{c}\text { Anzahlder } \\
\text { Gesichtsfelder } \\
\text { in einer } \\
\text { Präparateinheit. }\end{array}$ & $\begin{array}{l}\text { Länge des } \\
\text { Halbdurchmessers } \\
\text { in mm. }\end{array}$ & $\begin{array}{l}\text { Länge des } \\
\text { Durcll messers } \\
\text { in min. }\end{array}$ \\
\hline $\begin{array}{r}20 \\
30 \\
40 \\
45 \\
50 \\
55 \\
60 \\
65 \\
67 \\
70 \\
100 \\
200 \\
300 \\
400 \\
500 \\
600 \\
700 \\
800 \\
900 \\
1000 \\
1100 \\
1200 \\
1400 \\
1600\end{array}$ & $\begin{array}{l}1,26156 \\
1,030 \\
0,89206 \\
0,84104 \\
0,797883 \\
0,76076 \\
0,728,766 \\
0,69980 \\
0,698233 \\
0,674328 \\
0,564185 \\
0,398945 \\
0,32573 \\
0,282093 \\
0,25231 \\
0,23033 \\
0,21324 \\
0,199471 \\
0,18806 \\
0,178412 \\
0,17011 \\
0,16286 \\
0,15078 \\
0,14105\end{array}$ & $\begin{array}{l}2,523 \\
2,06 \\
1,784 \\
1,682 \\
1,596 \\
1,521 \\
1,457 \\
1,40 \\
1,378 \\
1,349 \\
1,128 \\
0,798 \\
0,651 \\
0,564 \\
0,505 \\
0,461 \\
0,426 \\
0,399 \\
0,376 \\
0.357 \\
0,34 \\
0,325 \\
0,302 \\
0,282\end{array}$ \\
\hline
\end{tabular}

Die Benutzung der Tabelle. Um irgendeinc ganze Zall der Gesichtsfelder zu erhalten, stellt man durch Auswahl des Objektivs und des Okulars und mittels Schraubenbewegung das Mikroskop so ein, daß dic Grenzen des Gesichtsfeldes am Durchmesser mit der der gewählten Zahl entsprechenden Teilstrichziffer des Kammerbodens zusammenfallen; z. B. Zahl 30 entspricht dem Durchmesser 2,06 mm Zahl $600-0,46 \mathrm{~mm}$ ete. $\mathrm{E}_{\mathrm{S}} \mathrm{muB}$ besonders beachtet werden, daB der Teilstrich mit dem Durchmesser des Gesichtsfeldes genau zusammenfalle. $\left.{ }^{1}\right)$ Sind die der gewählten Zahl entsprechenden Nummern des Objektivs und des Okulars und dic Tubuslänge des betreffenden Mikro skops bekannt, so braucht dic Durchinesserlänge des Gesichtsfeldes während der ganzen Dauer der Benutzung dieses Linsensystems nicht mehr bestimmt zu werden. Der Einfachheit wegen schlage ich vor, clie Anzahl der Gesichtsfelder in ciner Präparateinheit als Verhältnis in Form eines Bruches auszudrücken, z. B. 1/20,1/100 etc.

2. Dic Größe des Präparats oder das Volumen der Nornalkanmmel A beträgt $1 / 100 \mathrm{ccm}$, da diesc bei einer Fläche von $1 \mathrm{qcm}$ einc Tiefe von $1 / 10 \mathrm{mnl}(=1 / 100$ clil $)$ besitzt. Das Volumen der Arbeits kammer B beträgt $1 / 10 \mathrm{ccm}$, da ihre Fläche 4 qcon und ihre Tiefe ${ }^{1}, \mathrm{~m}$, d. l. ${ }^{1} / 40 \mathrm{~cm}$, ausmacht. Bei geringem Gehalt an Elementen inı Sediunent mul Kanmer B, bei großen Kammer A benutzt werden; ist die Zahl der Elemente sehr groß und das Sedinient selur gering, so $11 m$ lieses vordiinnt werden.

3. Die aus 100 ecm Harn gewonnene Menge des Sediments

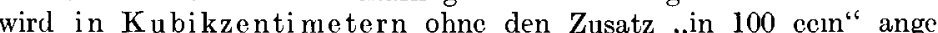
geben, da das selbstverständlicl ist. Vor der Untersuchung wird das Sediment durch Schütteln sorgfältig vermengt, weil sich die Eleinente beim Zentrifugicren schichtweise lagern, sich jedenfalls nicht regel mäßig verteilen. Je nachdem, ob das Sedinıent aus ïber oder unter $100 \mathrm{ecm}$ Harn gewonnen, oder ob es verdiinnt ist, niüssen entsprechende Berechnungen angestellt werden.

4. Um die Zahl der organisicrten Elemente im Gesichts. felde des Mikroskops zu bestimuen, tragen wir mittels einer wit ciner Gummikappe versehenen Pipette ein wenig Sedinent von bestimuter Größe auf den Boden der Normalkammer auf, sehieben vorsichtig das Deckgläschen herïber und zählen die Zylinder bei schwacher, dic Formelemente bei starker Vergrößerung. ${ }^{2}$ ) Um die Resultate der Zählungen möglichst fehlerfrei zu gestalten, inüssen allgemeingültige Regeln beobachtet werden: man hat dic Elemente unbedingt in vielen Gesichtsfeldern zu zählen und das arithmetische Mittel daraus zu nehınen;

$\left.{ }^{1}\right)$ Ich hatte anfänglich die Absicht, anf dem Kammerboden zwei Senkrechte mit Teilungen anbringen zu lassen, um gleichzeitig den Durchmesser bestimmen ind die Richtigkeit seiner Einstellung nachpräfen zu können. Das hätte aber die Kosten der Kammer beträchtlich erhöht.

2) Wenn man will, kann man auch Kristalle, insbesonderc die der Harnsäure und der Oxalate zählen, wobei ihre, wenn möglich durch Teilungen gemessene, vorherrschende Größe anzusehen ist. die sichtbaren Teile der Elenente ${ }^{1}$ ) in Betracht zu zielien; falls das Sediment in den Zwiselienraum zwischen dem Deckgläschen und dem Rahmen ecec dringt, ein ander(s Präparat anzufertigen etc.

Find alle erforderlichen Ziffern vorlaanden, so gestaltet sich dic Bcrechnung sehr cinfach.

Unı zu crfallen, wieviel Elemente in ciner Präparateinleit enthalten sind, inuß man die in cinem beliebigen Gesiclitsfelde der Kam ner A gefundene Zall der Flemente nit der Zall dieser in 1 qem entlaltenen Gesichtsfelder multipliziercn (bei Benutzung der Kamner B ist das Produkt durch 21, zu teilen), und zur Bestimmung der Elenente i gll gazen Sediment lat mall mir dic Zall der Slemente im Präparat mit der Zahl der il diescm Sediment cntlaltenen Präparate zu multiplizieren.

Zur Verdentlichung gestatte ich mir einige Beispicle anzufülıren.

1. Kanmer A, Sedinent 1,0 cem, 2 Zylinder in einem Gesichts. felde, dessen Fläche $1 / 60$ und 3 Erytlirozyten in einem Gesichtsfeldc. das $1 / 1200$ der Präparateinheit beträgt.

In Präparat werden $2 \times 60=120$ Zylinder und $3,1200=3600$ Erytlirozyten sein; das ganze Sediment wird $120 \because 100=12000 \mathrm{Zy}$ linder und $3600 \times 100=360000$ Erythrozyten enthalten.

2. Kammer B, 0,5 cem Sediment, 5 /ylinder in Gesichtsfolde $(1 / 5)$ und 10 Erythrozyten in Gesichtsfelde $(1 / 1000)$.

Da Kammer B 21/2mal tiefer ist als Kammer $A$, so werden in Präparat $-5 \frac{50}{2,5}=100$ Zylinder und $\frac{10 \cdot 1000}{2,5}=4000$ Erytlurozytel sein; das ganze Sediment wird 100 . 50 = 5000 Zylinder und $4000 \times 50$ $=200000$ Erythrozyten enthalten

3. Ist 0,22 cem Sediment vorlianden und sind in der gallzen Kamnner A 6 Zylinder gefunden, so werden inl ganzen Sediment $6 \times 22=132$ Zylinder enthalten sein, da das Volumen der Kainmer $A$ $1 / 100$ cem beträgt.

4. Ist 0,4 cem Sediment vorhanden und sind in der Kammer B nur 10 Zylinder gefunden, so wird dic Zahl der Zylinder in ganzen Sediment $10 \times 4=40$ betragen, da das Volmmen der Kanmer B $1 / 10 \mathrm{cem}$ ausmacht, und in der Präparateinheit wird 10:10=1 Zylinder enthalten sein.

Aus diesen wenigen Beispielen geht klar hervor, daß durcl dic Benutzung der Kammern und der Tabelle die quantitative Bestimunung der organisicrten Elemente des patlologischen Harnsediments bedeutend vercinfacht wird. Zwar crfordert dieses Verfahren etwas nıelır Zeit als die gewöhnlichen Analysen, dafür wird aber der Wirrwarr in der Untersuchungstechnik beseitigt und etwas Ordnung und Einheitlichkeit in das System der Harnanalyse gebracht, wodnrch es möglicl wird, der Wirklichkeit viel entsprechendere Ziffern zu erhalten.

Nur wenn eine allge meingültige Metlode der quantitative mikroskopischen Untersuchung des Harnsediments - sei es in vorliegender Form oder in anderer Gestalt - cingeführt würde, wär es möglich, ähnliche Kranke nach der Schwere der Nephritis zusammenzustellen, dic in verschicdenen Laboratorien ausgeführten Harnanalysen desselben Patienten zu vergleichen und - was das Wichtigste ist - den Verlauf der Nephritis an der Hand systematischer quantitativer Analysen, natürlicl im Verein mit allen anderen Merkmalen (Harnmenge, spezifisches Gewicht, Eiweißmenge, Oedem, Allgemeinbefinden...) zu verfolgen. $\left.{ }^{2}\right)$

In Anbetracht dessen, daß zur praktisehen Behandlung der Kraukeı und noch melir zur wissenseliaftlichen Beobachtung sowic zu experinien tellen Untersuchungen eine Einleitlicllkeit nikroskopiscliel Harnuntersucliungsmetloden unbedingtes Frfordernis ist, gostattc ich mir auf Grund obiger Darlegungen folgende Vorschläge:

1. Außer einer qualitativen unbedingt auch eine quantitative mikroskopische Untersuchung der Harnscdimente vorzunehinen;

2. zur qualitativen Analyse 24stündigen, zu systenatisehen vergleichenden quantitativen Untersuchungen aber frischen, unte genau denselben Bedingungen wälrend der acht, für del Schlaf bestion ten Stunden vou den Nicren abgesonderten Harn zu verwenden;

3. als Größeneinlueit des Sediments die aus 100 ccm frisclucn Harns durel Absetzen und Zentrifugieren gewonnene Menge festzusetzen dieses Sedimentquantuin in Kubikzentimetern anzugcben;

4. zu beständigen inikroskopisehen Untersuchungen des Sediments eine Ka m mer von 4 q $\mathrm{m}$ Fläcle und ${ }_{1}^{\prime}$ in m Ticfe, d. h. vou $1 / 10$ cem Volumen, zu akzepticren;

5. als Crößeneinleit des Präparats eine Harnzällkam mer von 1 qem Fläche und $1 / 10$ nt miefe, T. h. von $1 / 100$ ecm Volumen, anzuerkennen;

1) Um classalbe nicht zweimal zähles zu mürsen, kann ınan von den an der Kreislinie gelagerten Erytluozyten, Leukozyten, Nierenzellen und häufig auch Zyjindern ur die an der Linic eines der Halbkreise licgenden zählen. - 2) Auf colchell Beobachtungen basierende Arbeitan sind von mir in der Klinik des Herru Prof. Janowski aus. geführt worden und gelangen demnäehst zur Veröffentlichung. 
6. zu den Bercelnnungen dic auf dem Boden der Normalkammer angebrachten T'eilstriche und die Tabelle zu benutzen; und

7. die Zahl der organisierten Harnelemente in der Präparateinheit und hauptsächlich im ganzen Sediment zu be. stimnen; bei Berechnungen, welche sich nur auf cin Gesichtsfeld bezichen, das Verhältnis der Fläelıe dieses Gesichtsfeldes zur Präparatcinheit in Bruchform ( $1 / 20$ oder $1 / 1600)$ auszudrïcken.

Die Harnzählkanmmern (1. die kleine Kammer mit Teilungenl, 2. die kleine Kanmer ohne Teilungen, 3. die große Kammer ohne Teilungen) sind yon der Firma C. Zeiss, Jena, zu beziehen. 